\title{
Implantes de resina de poliuretana vegetal (Ricinus communis) na tração linear, fixação e fusão vertebral no cão. Estudo experimental
}

\author{
[Implants of Ricinus communis polyurethane resin in distraction, fixation and vertebral fusion in dogs. \\ Experimental study] \\ M.G. Laranjeira ${ }^{1}$, C.M.F. Rezende ${ }^{2}$, M.J.C. Sán ${ }^{3}$ C.M. Silva ${ }^{4}$ \\ ${ }^{1}$ Curso de Medicina Veterinária - Faculdade de Odontologia - UNESP \\ Rua Clóvis Pestana, 793, Bairro Dona Amélia \\ 16050-680 - Araçatuba, SP \\ ${ }^{2}$ Escola de Veterinária da UFMG \\ ${ }^{3}$ Aluno de Pós-Graduação da Escola de Veterinária da UFMG \\ ${ }^{4}$ Universidade Estadual do Ceará
}

\begin{abstract}
RESUMO
Placa e espaçador de polímero derivado do óleo de mamona (PDOM) (Ricinus communis) foram avaliados clínica, radiográfica e histologicamente na tração linear, fixação e fusão vertebral cervical em 20 cães adultos, sem raça definida, pesando entre 17 e $22 \mathrm{~kg}$. Foram sacrificados quatro animais aos 10 , 30, 60, 90 e 120 dias de pós-operatório. Após exposição da coluna cervical, por acesso ventral, o disco intervertebral de C4-C5 foi fenestrado e a abordagem ao canal medular foi feita por meio de fenda óssea. Um espaçador de PDOM foi colocado preenchendo o defeito ósseo. Os corpos vertebrais C4-C5 foram fixados com placa do mesmo material, utilizando-se dois parafusos corticais em cada corpo vertebral. Apenas um animal apresentou déficit neurológico no pós-operatório imediato. Radiograficamente as vértebras mostravam-se normais e alinhadas, sem colapso do espaço intervertebral, porém não houve neoformação óssea entre as vértebras. Ao exame mielográfico, não houve compressão da medula espinhal. Os implantes foram efetivos em manter a tração linear e fixação das vértebras cervicais e não ocorreu a fusão vertebral.
\end{abstract}

Palavras-chave: cão, coluna, implante, poliuretana

\begin{abstract}
Clinical, radiographic and histological evaluation of plate and biomechanical spacer of Ricinus communis polyurethane resin were performed in distraction, fixation and vertebral cervical fusion in 20 adult dogs, weighting between 17 and 22kg. Four animals were euthanized at 10, 30, 60, 90 and 120 days after surgery. After ventral exposition of cervical spine, cervical disk fenestration was performed on intervertebral spaces C4-C5 and a slot was created. A biomechanical spacer of Ricinus communis polyurethane resin was placed to fill bone defect. C4-C5 spinous processes were fixed with plate of the same material, utilizing two cortical screws in each spinal process. Only one animal had neurological signs immediately after surgery. Radiographic examination indicated that vertebrae were normal and aligned, without intervertebral colapse. However, there was no bone neoformation between C4-C5. The implants were effective to keep the distraction and fixation of cervical spine without vertebral fusion.
\end{abstract}

Keywords: dog, spine, implants, polyurethane

Recebido para publicação em 31 de janeiro de 2003

Recebido para publicação, após modificações, em 4 de fevereiro de 2004

E-mail: laranjei@fmva.unesp.br 


\section{INTRODUÇÃO}

A instabilidade da coluna cervical dos cães ocorre por diversas alterações tais como má formações, degenerações, fraturas, luxações e em decorrência de procedimentos descompressivos que levam à compressão da medula espinhal e das raízes nervosas.

Diferentes técnicas de distração, estabilização e fusão vertebral têm sido descritas no tratamento dessas alterações, seja por meio de imobilização externa ou pelo uso de implantes.

As placas plásticas muito utilizadas na estabilização da coluna cervical, como as placas Lubra (fluoreto de polinilideno) e de cloreto de polivinila (PVC), apresentam vantagens em relação aos métodos rígidos de fixação, por serem moldáveis e acompanharem os contornos vertebrais (Yturraspe e Lumb, 1972), no entanto, o alto custo das placas Lubra e, ainda, a possibilidade de efeito tóxico de algum material, como o PVC, muitas vezes impossibilitam o seu emprego (Arias, 2001).

As técnicas de distração e fusão vertebrais com emprego de espaçadores intervertebrais vêm sendo utilizadas, tanto na ortopedia humana, quanto na ortopedia veterinária. Para tanto, vários materiais têm sido utilizados, e o mais comum é o próprio osso. As limitações e dificuldades relativas aos enxertos ósseos autólogos, homólogos e heterólogos têm estimulado a pesquisa de substitutos ósseos para as mais diversas aplicações (Whitecloud III, 1999).

A pesquisa de biomateriais tem apresentado uma variedade de implantes sintéticos desenvolvidos para uso biológico. A descoberta de novos polímeros e copolímeros tem contribuído significativamente para a evolução no campo dos biomateriais. O Grupo de Química Analítica e Tecnologia de Polímeros - USP - São Carlos, desde 1984, desenvolve pesquisas com polímeros poliuretanos de óleo de mamona.

O polímero derivado do óleo de mamona é citado por seus múltiplos usos em medicina humana e veterinária. Vários são os relatos sobre o emprego desse produto como substituto do osso e de tecidos moles, e na forma de diferentes implantes para uso ortopédico e odontológico
(Caruzo e Roslindo, 1995; Carvalho et al., 1997; Fernandes, 2000; Ignácio et al., 2002). Por ser um produto nacional, de baixo custo, considerado atóxico e biocompatível, seu emprego é de grande utilidade em medicina veterinária, onde os custos, muitas vezes, impedem uma intervenção.

Dessa forma, o objetivo deste trabalho foi avaliar clínica e radiograficamente o emprego de espaçador e placa de polímero derivado do óleo da mamona (Ricinus communis) com $30 \%$ de carbonato de cálcio na tração linear/estabilização e fusão vertebrais.

\section{MATERIAL E MÉTODOS}

Foram utilizados 20 cães adultos, com idade entre dois a quatro anos, sem raça definida, de ambos os sexos, e pesos entre 17 e $22 \mathrm{~kg}$. Durante o período de 15 dias de adaptação os animais foram mantidos em canis individuais, tratados com ração balanceada ${ }^{1}$ e água ad libitum. Nesse período foram submetidos a exames clínico e neurológico. Procedeu-se à colheita de material para exames de laboratório (hemograma e leishmaniose) e radiográficos simples da coluna cervical, para verificação de alterações que pudessem interferir com o experimento. Os animais foram distribuídos aleatoriamente em cinco grupos, com quatro animais cada.

Os espaçadores foram elaborados com medidas padrão de quatro milímetros de largura, $6 \mathrm{~mm}$ de profundidade e $15 \mathrm{~mm}$ de comprimento. As placas foram cortadas em forma de $\mathrm{H}$ a partir de uma chapa com $3,6 \mathrm{~mm}$ de espessura, com largura de $18 \mathrm{~mm}$ e comprimento variando de $45 \mathrm{~mm}$ a $78 \mathrm{~mm}$.

Após jejum sólido e hídrico de 12 horas, os animais foram submetidos à medicação préoperatória que constou da administração de cefalotina sódica ${ }^{2}(30 \mathrm{mg} / \mathrm{kg}$ IV) 30 minutos antes da cirurgia. Após serem pré-anestesiados com cloridrato de xilazina $(1 \mathrm{mg} / \mathrm{kg} \mathrm{IV})^{3}$ por via intravenosa, realizou-se a tricotomia da região ventral da coluna cervical. A indução anestésica foi feita com tiopental sódico $(12,5 \mathrm{mg} / \mathrm{kg} \mathrm{IV})^{4} \mathrm{e}$,

${ }^{1}$ Pedigree Champ - Éffem

${ }^{2}$ Cefalotina sódica - Eurofarma Laboratório Ltda

${ }^{3}$ Rompum $2 \%$ - Bayer S.A.

${ }^{4}$ Thionembutal sódico - Abbott Lab. do Brasil 
após entubação endotraqueal, a manutenção anestésica com halotano 5 .

Após o acesso ventral à quarta e quinta vértebras cervicais, realizado segundo técnica descrita por Wheeler e Sharp (1999), colocou-se um distrator modificado de Herrington entre os processos transversos esquerdos da quarta e quinta vértebras cervicais, exerceu-se tração até a imobilização dessas vértebras, e realizou-se a fenestração do disco intervertebral entre C4-C5.

Empregando-se broca para osso, acoplada a motor pneumático de alta rotação ${ }^{6}$, foi realizado o acesso ventral ao canal medular por meio de uma fenda óssea (slot) retangular na região ventral dos corpos vertebrais de C4-C5, tomando-se os cuidados em manter a sua linha média e não lesionar a artéria e o seio vertebrais. Esse procedimento foi realizado sob irrigação constante com solução fisiológica ${ }^{7}$ a $0,9 \%$. A extensão do defeito foi de $4 \mathrm{~mm}$ de largura e $15 \mathrm{~mm}$ de comprimento e a profundidade foi determinada pelas visualização das três camadas distintas do osso.

Após a remoção da cortical interna, foi retirada a porção dorsal do anel fibroso com auxílio de lâmina de bisturi $\mathrm{n}^{\circ} 11$ e mantidas as áreas laterais do disco intervertebral.

Realizada a fenestração do disco intervertebral (DIV) e o acesso ao canal medular, o defeito ósseo foi preenchido com um espaçador de polímero derivado do óleo de mamona (PDOM) com carbonato de cálcio a $30 \%$. O distrator foi então removido e uma placa de PDOM com carbonato de cálcio a $30 \%$ foi aplicada sobre o quarto e quinto corpos vertebrais cervicais. Antes da aplicação a placa foi aquecida em solução fisiológica estéril entre $70^{\circ} \mathrm{C}$ e $80^{\circ} \mathrm{C}$, para moldagem e adaptação aos contornos ósseos. Foram feitos quatro orifícios em cada placa, com broca $3,5 \mathrm{~mm}$. As placas foram fixadas sobre a superfície ventral da quarta e quinta vértebras cervicais com parafusos ortopédicos do tipo cortical com diâmetro de $3,5 \mathrm{~mm}$. Os orifícios foram feitos com broca de $2,5 \mathrm{~mm}$ e furadeira pneumática orientados ventrolateralmente em um

${ }^{5}$ Halothano - Cristália Produtos Químicos Farmacêuticos Ltda

${ }^{6}$ Furadeira pneumática $-3 \mathrm{M}$.

Solução fisiológica de cloreto de sódio (isotônica) -

Brasmédica S/A Indústrias Farmacêuticas. ângulo de cerca de $30^{\circ}$ a $35^{\circ}$ em relação à vértebra. Após a utilização do macho de $3,5 \mathrm{~mm}$ para confecção da rosca, os parafusos foram colocados na seguinte seqüência: orifício caudal esquerdo, orifício cranial direito e vice-versa, até a completa fixação da placa.

Após a irrigação dos tecidos com solução fisiológia a $0,9 \%$, a síntese dos tecidos foi feita de forma rotineira. Durante os 10 primeiros dias de pós-operatório, a ferida cirúrgica foi protegida com gaze e algodão ortopédico e a região cervical foi envolvida com atadura de crepom. Foi administrado antiinflamatório não esteróide, vedaprofen $^{8}$, na dose de $0,5 \mathrm{mg} / \mathrm{kg}$, em todos os animais, por cinco dias. O acompanhamento clínico e neurológico foi feito diariamente nos primeiros 10 dias de pós-operatório e em intervalos quinzenais até o dia da eutanásia. Durante os 10 primeiros dias, o exame neurológico foi restrito à avaliação dos reflexos espinhais nos membros torácico e pélvico, à alterações na marcha e sensibilidade dolorosa, superficial e profunda, e à sensibilidade cervical por meio de extensão e flexão do pescoço.

Os animais foram submetidos a controles radiográficos simples, nas incidências laterolateral e ventrodorsal nos períodos pósoperatório imediato, 10, 30, 60, 90 e 120 dias de pós-operatório. $\mathrm{O}$ exame mielográfico foi realizado após as radiografias simples. A avaliação radiográfica simples objetivou verificar a integridade do espaço intervertebral no sítio operado e dos espaços intervertebrais adjacentes, o alinhamento das vértebras operadas, o posicionamento da placa, dos parafusos e do espaçador intervertebral, e a reação óssea local.

\section{RESULTADOS E DISCUSSÃO}

A bandagem aplicada na região cervical permaneceu até a retirada dos pontos, aos 10 dias de pós-operatório, e foi efetiva no controle da formação de seroma, com exceção de um cão, cuja faixa foi removida após 24 horas.

Apenas um animal apresentou dificuldade de deambulação, com ausência de propriocepção do membro torácico esquerdo e sinais de dor à manipulação cervical. $\mathrm{O}$ déficit neurológico

${ }^{8}$ Quadrisol 5 - Intervet 
observado logo após o retorno anestésico, e que regrediu oito dias após a cirurgia, foi provavelmente atribuído à intensidade de tração linear. Nesse animal, foi utilizado um distrator com garras maiores e a força aplicada também foi de maior intensidade. Os sinais neurológicos foram apenas no membro torácico esquerdo, lado de aplicação do distrator, sugerindo alguma lesão na raiz nervosa (Wheeler e Sharp, 1999). Nenhum trauma perceptível foi provocado à medula espinhal durante a realização do defeito ósseo ou na colocação dos parafusos.

As radiografias simples laterolateral e ventrodorsal da coluna cervical, no pós- operatório imediato, mostraram que as vértebras apresentavam-se normais e alinhadas (Fig. 1 e 2). $\mathrm{Na}$ projeção laterolateral, nos locais em que a placa não apresentava contato com os corpos vertebrais, verificou-se o distanciamento de cerca de $1-2 \mathrm{~mm}$ entre a placa e o corpo vertebral (Fig. 1), não ocorrendo afastamento das placas em relação aos corpos vertebrais nos períodos subseqüentes. A distância entre as placas e os corpos vertebrais, evidenciada na avaliação radiográfica, deve ser considerada menor, pois segundo Owens (1982), dependendo da técnica radiográfica utilizada, o tamanho real de uma estrutura pode ser ampliado.

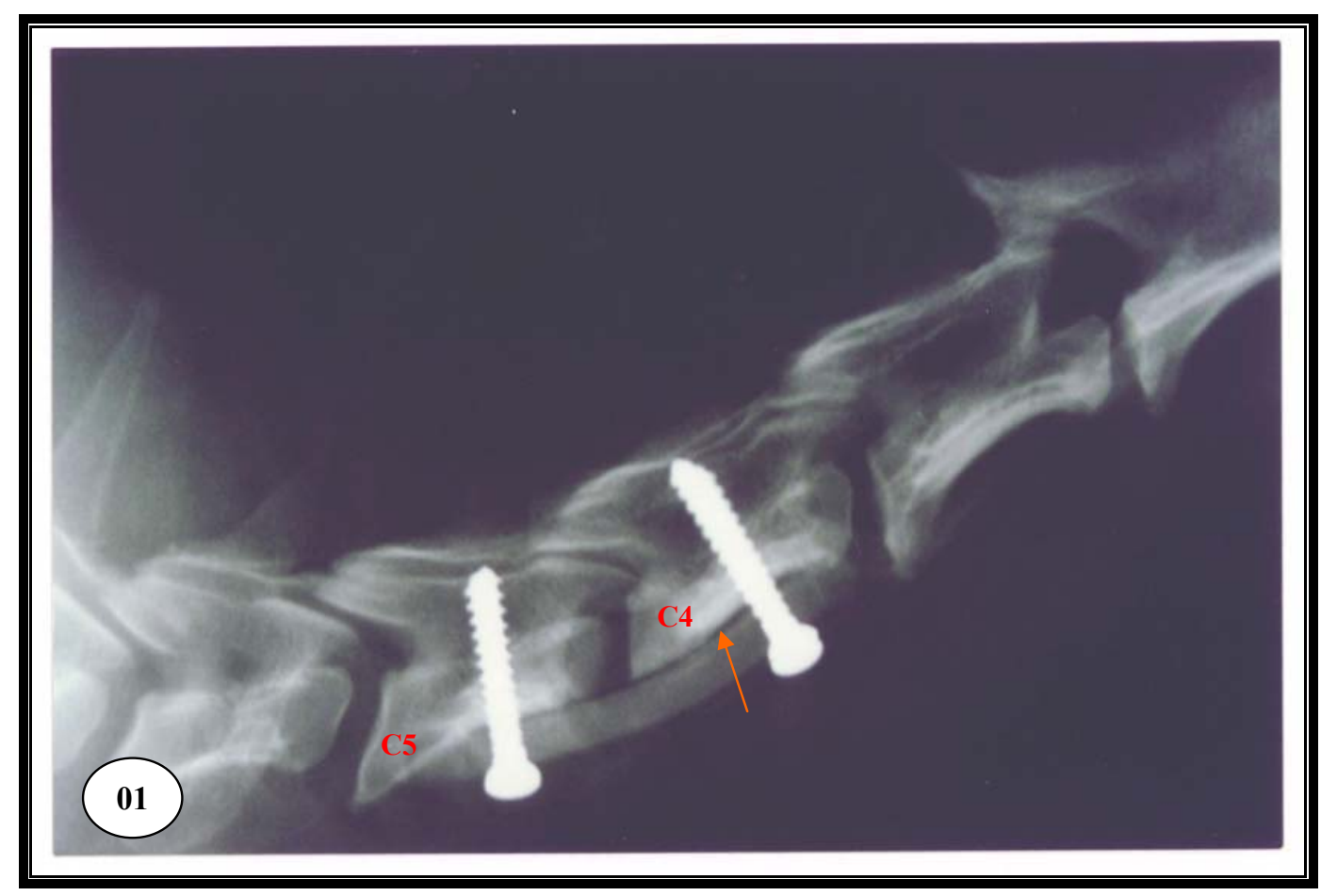

Figura 1. Radiografia simples laterolateral da coluna vertebral cervical, pós-operatório imediato. Observar o alinhamento das vértebras operadas, o distanciamento entre placa e corpo vertebral (seta). 


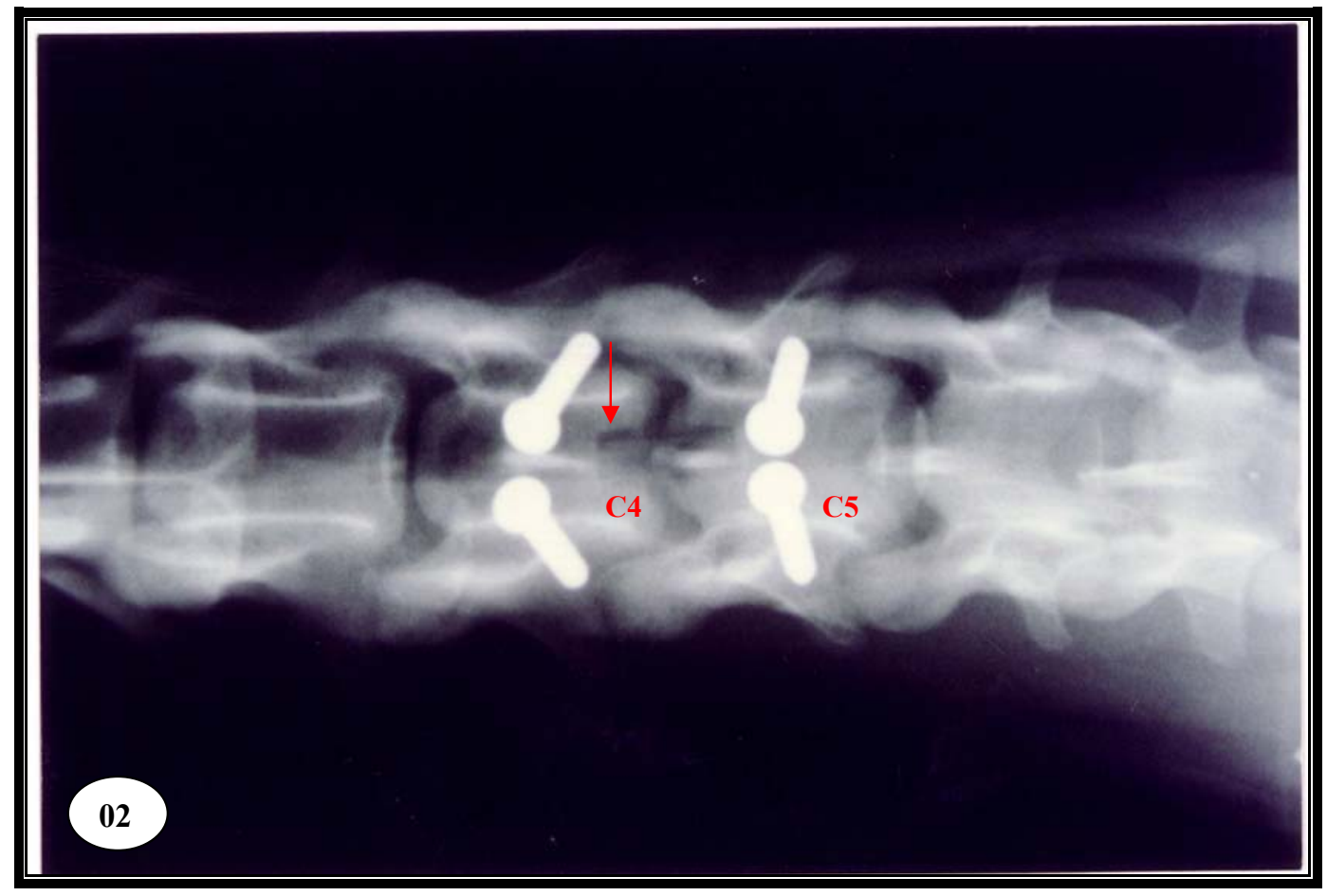

Figura 2. Radiografia simples ventrodorsal da coluna vertebral cervical, pós-operatório imediato. Observa-se a angulação dos parafusos em relação aos corpos vertebrais. Halo radioluscente entre espaçador e osso (seta).

A projeção ventrodorsal mostrou a angulação dos parafusos em relação ao corpo vertebral, mas não permitiu afirmar se houve ou não a penetração do canal medular (Fig. 2). Houve migração total de um parafuso em um cão do grupo IV (caudal direito) e parcial em um cão do grupo III (caudal esquerdo), observada aos 30 dias de pósoperatório. A migração dos parafusos pode ocorrer por várias razões, como o uso de furadeira com rotação muito alta, a constituição das vértebras cervicais, o emprego de parafusos com diâmetro inadequado, a imobilização externa por curto período de tempo, a rejeição ao implante e o excesso de tensão no parafuso caso não ocorra boa acomodação da placa (Smith e Walter, 1985; Boudrieau, 1991; Jeffery, 1995; Bruecker, 1996). Neste experimento, a ausência de contato entre placa e osso não interferiu com a imobilização vertebral ou com a migração dos parafusos pois, segundo Conzemius e Swainson (1999), as placas que seguem o contorno ósseo asseguram, pelo menos, $30 \%$ de contato entre placa e osso, diminuindo as forças de tensão sobre os parafusos A migração dos parafusos, neste trabalho, foi em decorrência de erro de técnica, fato observado em dois cães. $\mathrm{Na}$ tentativa de correção do ângulo de perfuração com a broca, houve desgaste maior do osso, ampliando o diâmetro do orifício. No entanto, esse fato não comprometeu o método de fixação.

A protrusão do disco intervertebral cranial às vértebras operadas foi observada em dois cães, $10 \%$ dos casos, aos 30 dias de pós-operatório, porém sem ocorrência de sinais neurológicos. A fusão de um espaço intervertebral aumenta o estresse no disco intervertebral adjacente, podendo levar à degeneração crônica do DIV e à sua instabilidade ou acelerar um processo degenerativo subclínico, complicação denominada efeito dominó. Isso ocorre em cerca de $25 \%$ dos cães entre o quinto e o $60^{\circ}$ mês de pós-operatório (Bruecker et al., 1989). A ocorrência aguda da protrusão do disco 
intervertebral encontrada neste trabalho difere dos relatos anteriores. A degeneração dos discos intervertebrais implica em cronicidade. Segundo Lincon e Petit (1985), os DIV de cães sujeitos à artrodese vertebral sofrem alterações no metabolismo, mas são capazes de acomodar essa hiperatividade metabólica, sem evidências de degeneração. $\mathrm{O}$ curto período entre a cirurgia e a observação do efeito dominó parece suportar a teoria da coexistência de lesão subclínica nos discos adjacentes. Nesses animais não foram observadas alterações radiográficas dos DIV no período pré-operatório, no entanto os resultados não excluem a possibilidade de lesão subclínica (Wilson et al., 1994).

Não se observou reação óssea sob a placa. Contudo, entre o espaçador e o osso foi verificado um halo radioluscente na avaliação ventrodorsal imediata ao ato cirúrgico (Fig. 2). Nas avaliações subseqüentes o halo permaneceu e, em cinco animais, observou-se aumento aos 30 dias de pós-operatório, que se manteve sem alterações até as avaliações finais.

A presença constante de radioluscência no local do DIV indicou a não reparação óssea esperada unindo os corpos vertebrais. Apesar desse material não apresentar radiopacidade que impeça a visualização da reparação óssea, neste trabalho a avaliação da reparação óssea dorsal ao espaçador foi subjetiva, pela sobreposição das estruturas ósseas. Nenhum animal apresentou colapso do espaço intervertebral C4-C5.

A mielografia confirmou a não compressão da medula espinhal no local da cirurgia, tanto na projeção laterolateral quanto ventrodorsal, e não se verificou desvio ou estreitamento das colunas de contraste nos dois cães que apresentaram estreitamento do terceiro espaço intervertebral cervical (Fig. 3 a e b).

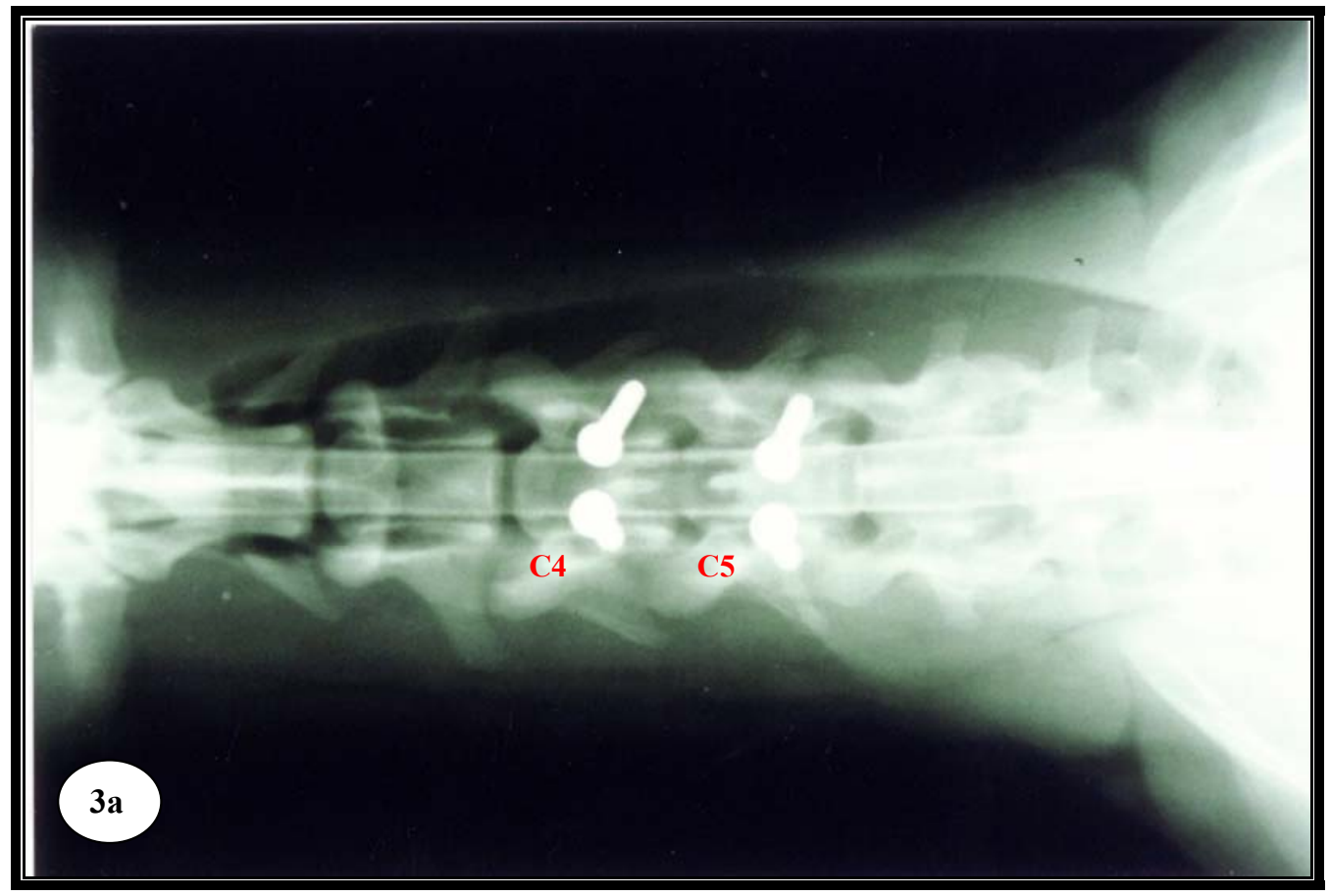




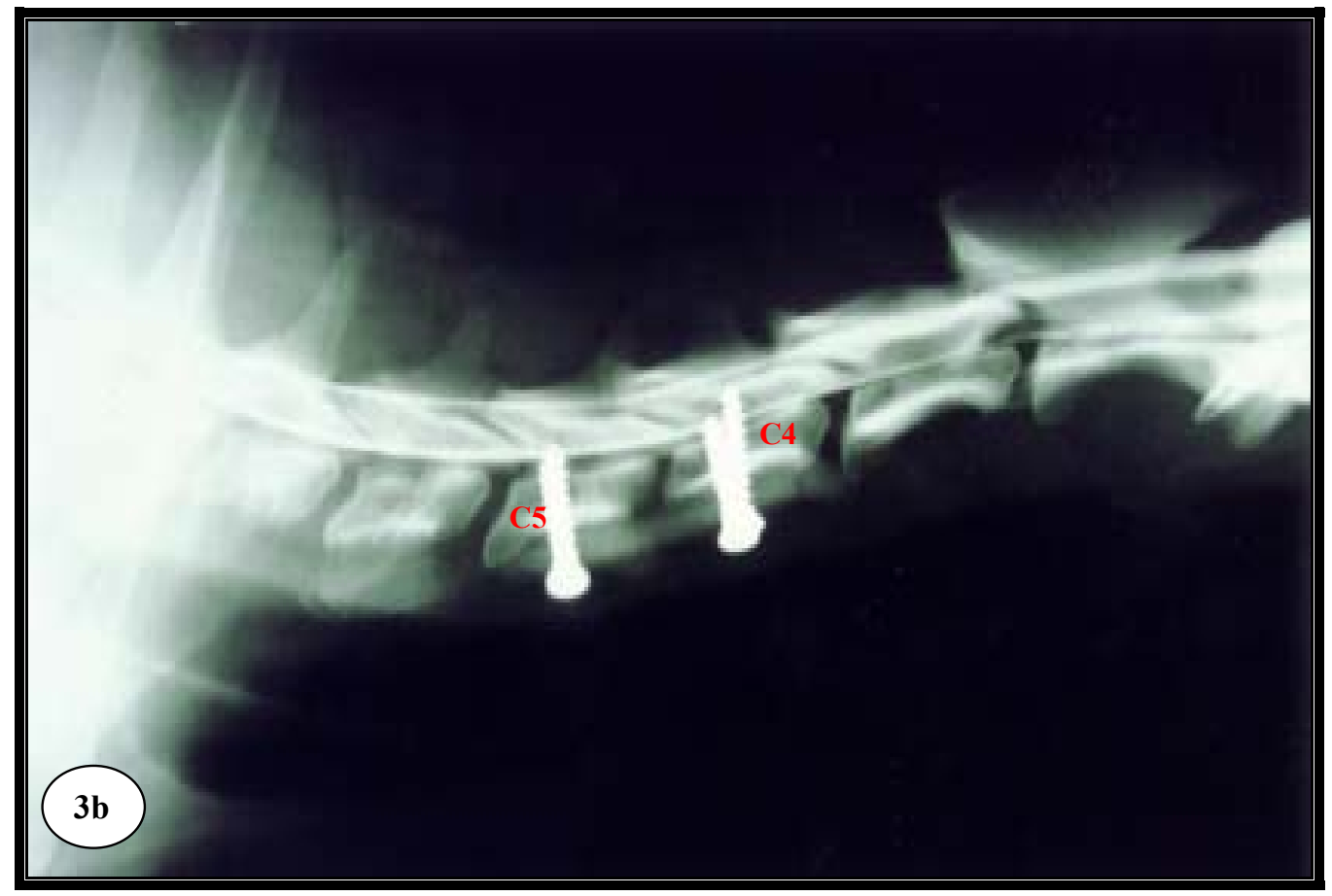

Figura 3. Mielografias da coluna vertebral cervical aos 120 dias de pós-operatório. Observa-se ausência de alterações nas linhas de contraste em todo o segmento cervical (A - incidência ventrodorsal, B incidência laterolateral).

\section{CONCLUSÕES}

Nas condições do presente estudo e com base nos resultados obtidos, pôde-se concluir que o espaçador e a placa de PDOM foram clínica e radiograficamente eficientes em promover a tração linear e a estabilização da coluna cervical, porém não houve fusão vertebral.

\section{REFERÊNCIAS BIBLIOGRÁFICAS}

ARIAS, M.V.B. Estudo experimental sobre a estabilização da coluna vertebral cervical caudal de cães com placas de cloreto de polivinila. 2001. 85f. Tese (Doutorado em Cirurgia)- Faculdade de Medicina Veterinária e Zootecnia, Universidade de São Paulo, São Paulo.

BOUDRIEAU, R. Principles of screw and plate fixation. Sem. Vet. Med. Surg. Small Anim., v.6, p.75-89, 1991.
BRUECKER, K.A.; Principles of vetebral fracture management. Sem. Vet. Med. Surg. Small Anim., v.11, p.259-272, 1996.

BRUECKER, K.A; SEIM, H.B.; BLASS, C.E. Caudal cervical spondylomyelopathy: decompression by linear traction and stabilization with steinmann pins and polymethyl methacrylate. J. Am. Anim. Hosp. Assoc., v.25, p.677-683, 1989.

CARUZO, S.L.; ROSLINDO, N.C. Implantes de resina poliuretana vegetal em arco zigomático de ratos. Estudo histológico. In.: JORNADA FARMACÊUTICA DE ARARAQUARA, 9., 1995, Araraquara. Resumos... Araraquara: Faculdade de Ciências Farmacêuticas, 1995. P.116.

CARVALHO, T.L.; ARAÚJO, C.A.C.A.; TEÓFILO, J.M. et al. Histologyc and hityometric evalualtion of rat alveolar wound healing around polyurethane resin implants. Int. J. Oral Maxilofac. Surg., v.26, p.149-152, 1997. 
CONZEMIUS, M.; SWAINSON, S. Fracture fixation with screws and bone plates. Vet. Clin. North Am.: Small Anim. Pract., v.29, p.11171133, 1999.

FERNANDES, E.S. Estudo experimental do emprego do polímero derivado do óleo de mamona (Ricinus communis) na diáfise proximal da tíbia de cães. 2000. 36f. Dissertação (Mestrado)- Universidade Federal de Viçosa, Viçosa, MG.

IGNÁCIO, H.; MAZZER, N.; BARBIERI, C.H. et al. Utilização da poliuretana da mamona nas formas compacta e porosa no preenchimento de falha óssea: estudo experimental em cães. Rev. Bras. Ortop., v.37, p.187-194, 2002.

JEFFERY, N.D. Cervical spinal surgery. In.: JEFFERY, N.D. Handbook of small animal spinal surgery. London: W. B. Saunders, 1995. P.148-166.

LINCON, J.D.; PETTIT, G.D. Evaluation of fenestration for treatment of degenerative disc disease in the caudal cervical region of large dogs. Vet. Surg., v.14, p.240-246, 1985.
OWENS, J.M. Radiografic interpretation for the small animal clinician. Saint Louis: Ralston Purina, 1982. 207p.

SMITH, G.K.; WALTER, M.C. Fractures and luxation of the spine. In: NEWTON, C.D.; NUNAMAKER, D.M. Texbook of small animal orthopaedics. Philadelphia: Lippincot, 1985. P.307-322.

WHEELER, S.J.; SHARP, N.J.H. Diagnóstico e tratamento cirúrgico das afecções espinhais do cão e do gato. São Paulo: Manole, 1999. 224p.

WHITECLOUD III, T. Modern alternatives and techniques for one-level discectomy and fusion. Clin. Orth. Rel. Res., v.359, p.67-76, 1999.

WILSON, E.R.; ARON, D.N.; ROBERTS, R.E. Observation of a secundary compressive lesion after treatment of caudal cervical spondylomyelopathy in a dog. J. Am. Vet. Med. Assoc., v.205, p.1297-1299, 1994.

YTURRASPE, D.J.; LUMB, W.V. The use of plastic plates for internal fixation of the canine spine. J. Am. Vet. Med. Assoc., v.161, p.16511657, 1972. 